\title{
Caracterización de pacientes con tumores neuroendocrinos en un hospital de referencia de alta complejidad
}

\author{
Characterization of patients with neuroendocrine tumors in \\ a referral hospital
}

\author{
Natalia Flórez $\mathbb{D D}^{\mathbb{D}}$, Juan Camilo Pérez², Álvaro Turizo ${ }^{\mathbb{D}}$, Diana Paola Cuesta ${ }^{4} \mathbb{D}$ \\ Médica, especialista en Cirugía General, Universidad Pontificia Bolivariana, Medellín, Colombia. \\ Médico, especialista en Patología, Hospital Pablo Tobón Uribe, Medellín, Colombia. \\ Médico, especialista en Cirugía General, Universidad Pontificia Bolivariana, Medellín, Colombia. \\ Médica, PhD en Epidemiología, Facultad de Medicina, Universidad Pontificia Bolivariana, Medellín, Colombia.
}

\section{Resumen}

Introducción. Los tumores neuroendocrinos comprenden una amplia familia tumoral, siendo de mayor frecuencia aquellos localizados en el tracto gastrointestinal, los pulmones, el timo y el páncreas. Ocurren con poca frecuencia y cursan con un comportamiento biológico variable. El objetivo del presente estudio fue describir las características demográficas y clínicas de los pacientes con esta entidad en un hospital de alta complejidad.

Métodos. Estudio descriptivo con revisión de historias clínicas correspondientes a pacientes con tumor neuroendocrino, cuya muestra histológica fue analizada en el Departamento de Patología del Hospital Pablo Tobón Uribe, Medellín, Colombia, entre 2010 y 20I5. Se describen frecuencias de localización, grado de diferenciación y perfil de inmuno-histoquímica.

Resultados. Se revisaron III historias, de las que el 5I,5\% correspondió a pacientes masculinos, con una mediana de edad al momento del diagnóstico de 48 años. Las comorbilidades más frecuentes fueron: hipertensión arterial $(38,6 \%)$, hipotiroidismo ( $15,8 \%$ y diabetes mellitus (II,9\%). El antecedente de neoplasia estuvo presente en el I3,9 \% de los casos. Un 59,4 \% de las muestras correspondieron a tumores primarios, el 34,8 \% estaban localizados en el páncreas. El hígado fue el órgano más comprometido por metástasis (73,3\%).

De los tumores neuroendocrinos, el $27 \%$ correspondió a tumores grado I del tracto gastrointestinal o del páncreas, el $36 \%$ a grado 2 y el $27,9 \%$ a grado 3 . El 3,6 \% correspondió a tumores pulmonares de grado bajo o intermedio y el $5,4 \%$ a tumores pulmonares de alto grado. Los marcadores tumorales con mayor positividad fueron sinaptofisina (97,2 \%), citoqueratinas $\mathrm{AEI} / \mathrm{AE} 3(95 \%), \mathrm{CD} 56(91,3 \%)$ y cromogranina $(87,8 \%)$.

Fecha de recibido: 02/05/2019 - Fecha de aceptación: 30/01/2020

Correspondencia: Álvaro Turizo Agámez. Calle 78B \# 72A-109, Medellín, Colombia. Código postal 050034. Teléfono: 3006593186 Correo electrónico: alvaro2014@hotmail.com

Citar como: Flórez N, Pérez JC, Turizo A, Cuesta DP. Caracterización de pacientes con tumores neuroendocrinos en un hospital de referencia de alta complejidad. Rev Colomb Cir. 2020;35:639-46. https://doi.org/10.30944/20117582.786

Este es un artículo de acceso abierto bajo una Licencia Creative Commons - BY-NC-ND https://creativecommons.org/licenses/by-ncnd/4.0/deed.es 
Discusión. Los tumores neuroendocrinos tienen características clínicas e histopatológicas diversas. Conocer las características de los pacientes afectados permite una aproximación de la epidemiología local aplicable a futuras investigaciones.

Palabras clave: carcinoma neuroendocrino; tumor carcinoide; sistema neurosecretor; diagnóstico; inmunohistoquímica; clasificación del tumor; estadificación de neoplasias.

\begin{abstract}
Introduction. Neuroendocrine tumors comprise a broad family of tumors, the most common being those located in the gastrointestinal tract, lungs, thymus, and pancreas. They occur infrequently and have a variable biological behavior. The objective of this study was to describe the demographic and clinical characteristics of patients with this entity in a highly complex hospital.
\end{abstract}

Methods. Descriptive study with review of medical records corresponding to patients with neuroendocrine tumors, whose histological sample was analyzed in the Pathology Department of the Pablo Tobón Uribe Hospital in Medellín between 2010 and 2015. Frequencies of location, degree of differentiation and immune-histochemistry profile are described.

Results. One hundred eleven histories were reviewed, of which $51.5 \%$ corresponded to male patients, with a median age at diagnosis of 48 years. The most frequent comorbidities were arterial hypertension (38.6\%), hypothyroidism (15.8\%) and diabetes mellitus (II.9\%). Medical history of neoplasia was present in $13.9 \%$ of the cases. $59.4 \%$ of the samples corresponded to primary tumors, $34.8 \%$ were located in the pancreas. The liver was the organ most compromised by metastasis (73.3\%). Of the neuroendocrine tumors, $27 \%$ corresponded to grade I tumors of the gastrointestinal tract or pancreas, $36 \%$ to grade 2 and $27.9 \%$ to grade $3 ; 3.6 \%$ corresponded to low or intermediate grade lung tumors and $5.4 \%$ to high-grade lung tumors. The tumor markers with the highest positivity were synaptophysin (97.2\%), cytokeratins AEI/AE3 (95\%), CD56 (9I.3\%) and chromogranin (87.8\%).

Discusion. Neuroendocrine tumors have diverse clinical and histopathologic features. Knowing the characteristics of affected patients allows an approximation of local epidemiology applicable to future research.

Keywords: neuroendocrine carcinoma; carcinoid tumor; neurosecretory system; diagnosis; immunohistochemistry; grading neoplasm; neoplasm staging.

\section{Introducción}

El término "neuroendocrino" describe un tipo celular que tiene características morfológicas y fisiológicas tanto del sistema endocrino como del sistema nervioso, en forma simultánea ${ }^{\text {I. El }}$ sistema neuroendocrino está constituido por dos componentes, uno sólido o glandular, conformado por la glándula pituitaria, las paratiroides y la médula adrenal, y otro difuso que comprende células distribuidas en la piel, la tiroides, los pulmones, el timo, el páncreas, el tracto gastrointestinal, la vía biliar y el tracto urogenital. La mayor proporción de células de este sistema se encuentran en el tracto gastrointestinal, y por esto se considera como el órgano endocrino más grande de todo el cuerpo humano. La principal característica que comparten las células neuroendocrinas es la producción de una amplia variedad de aminas y polipéptidos, involucrados en la homeostasis y la regulación de diversos procesos endocrinos.

Los Tumores Neuroendocrinos (TNE) son neoplasias raras, derivadas del sistema que da origen a su nombre. Según estadísticas nacionales, en un estudio realizado en el Instituto Nacional de Cancerología, Bogotá, Colombia, 
entre 1988 y 1992 se encontraron tan solo 26 ca$\operatorname{sos}^{2}$. Con datos un poco más recientes, se conoce que la incidencia de esta entidad ha presentado un incremento estadístico importante, aun así, los tumores neuroendocrinos corresponden tan solo al $2 \%$ de todas las neoplasias del tracto gastrointestinal con potencial maligno, según un consenso latinoamericano que contó con la participación de expertos colombianos 3 .

Los TNE pueden aparecer en diversos tejidos corporales, incluso en el epitelio respiratorio. Una de las clasificaciones iniciales de los TNE, tenía en cuenta el origen embriológico de la estructura afectada, dadas algunas similitudes en el comportamiento y la topografía de dichas lesiones, siendo entonces agrupadas en neoplasias de intestino anterior (pulmón, bronquio, estómago), intestino medio (intestino delgado, apéndice cecal y colon proximal) e intestino posterior (colon distal y recto) ${ }^{4}$. Sin embargo, ante el surgimiento de diversos sistemas de estadificación, y en aras de evitar la confusión a que pudiera dar lugar, se estableció como norma internacional el uso de la clasificación de tumores neuroendocrinos propuesta por la Organización Mundial de la Salud (OMS) en 20Io, la cual señala tres grupos: tumores bien diferenciados o grado I, moderadamente diferenciados o grado II y mal diferenciados o grado III, de acuerdo con el grado de proliferación celular (medida con el índice Ki-67) y con el conteo de mitosis de la muestra estudiada 5 .

La presentación clínica de los TNE suele ser variable, desde pacientes asintomáticos en quienes se realiza el diagnóstico en forma incidental, hasta la presencia de síntomas difusos y totalmente inespecíficos que generan retrasos en el enfoque y manejo adecuado de estos enfermos. Usualmente los síntomas se relacionan con la localización del tumor o con el desarrollo del conocido "síndrome carcinoide", un conjunto de expresiones sistémicas generadas por la producción de sustancias neuroendocrinas que, liberadas al torrente circulatorio pueden desencadenar diarrea, pérdida de peso, diaforesis y alteraciones cardiovasculares entre otras.
La probabilidad de compromiso metastásico se ha asociado al sitio del tumor primario, de hecho, se conoce que es más común en pacientes con tumores de intestino delgado que cuando se presenta en otra localización, encontrando que hasta el $60 \%$ de estos pacientes tienen compromiso nodal en el momento del diagnóstico inicial ${ }^{6}$. Idealmente, los pacientes afectados por TNE, deben ser tratados por un grupo multidisciplinario, dado que, si bien la cirugía es la única opción curativa en los tumores localizados y ciertos tumores metastásicos que presentan lesiones primarias y secundarias resecables, en los pacientes con enfermedad irresecable, el tratamiento médico está recomendado e incluye el uso de análogos de la somatostatina, interferón alfa, terapia radio-nuclear y más recientemente, terapias genéticamente dirigidas ${ }^{7.8}$. En Colombia, no existen guías específicas para el enfoque diagnóstico y terapéutico de estas lesiones, por lo tanto, el manejo de estos pacientes se realiza con base en consensos y guías internacionales, extrapolando los resultados y las recomendaciones de esta evidencia actual.

Esta investigación pretende describir las principales características demográficas y clínicas de los pacientes diagnosticados en el departamento de patología de un hospital de alta complejidad y centro de referencia colombiano. Se espera que estos resultados contribuyan a comprender la evolución clínica y el abordaje de estos pacientes, lo que se considera un aporte importante, ante la ausencia de estudios locales similares.

\section{Métodos}

Se realizó un estudio observacional descriptivo de pacientes con tumores neuroendocrinos, primarios y metastásicos, diagnosticados por histología en el Departamento de Patología del Hospital Pablo Tobón Uribe, Medellín, Colombia, en el periodo 20I0-2015. Se excluyeron aquellos pacientes con información incompleta en la historia clínica y los pacientes remitidos a esta institución con el diagnóstico histológico ya establecido. 
Las fuentes de información fueron los informes de patología y las historias clínicas de las cuales se obtuvieron las variables a analizar: características sociodemográficas (género, edad al momento del diagnóstico), comorbilidades (hipertensión arterial, hipotiroidismo, enfermedad coronaria, fibrilación auricular y dislipidemia, entre otras), el antecedente o diagnóstico actual de neoplasias y su localización. Con respecto a los tumores, se estudiaron las siguientes variables clínicas: localización del tumor, tipo de lesión (primaria o metastásica), grado de diferenciación histológica según la Organización Mundial de la Salud (OMS) 20Io, de acuerdo con el marcador Ki-67 y el número de mitosis por campo de alto poder (para los tumores neuroendocrinos gastrointestinales o pancreáticos) y la positividad de los marcadores de inmunohistoquímica (IHQ).

En el caso de los tumores del tracto respiratorio, se agruparon según la subclasificación de la OMS para este tipo específico de neoplasias en: grado bajo/intermedio si correspondían a carcinoide típico y atípico y alto grado si su principal característica fue la presencia de células grandes y pequeñas.

Se diseñó un formato para la recolección de la información en Excel y posteriormente se exportó la base de datos a IBM SPSS Statistics 22 para el análisis de las variables descritas. Las variables categóricas se presentaron como frecuencias absolutas y relativas, y las continuas como rango, mediana y rango intercuartílico.

\section{Resultados}

Durante el periodo de estudio se identificaron 125 muestras de pacientes con diagnóstico de TNE en la institución. Se excluyeron I4 muestras (cinco por registros incompletos en la historia clínica, ocho sin reporte de inmunohistoquímica y uno por diagnóstico de hiperplasia del páncreas), y finalmente se analizaron III pacientes.

\section{Características clínicas}

Un 5I,5\% correspondieron a hombres, el rango de edad de los pacientes al momento del diagnóstico fue entre I4 y 82 años, con una mediana de
48 años (RIQ: 50 - 67). Un 6o,4\% de los pacientes presentaron algún tipo de comorbilidad, siendo las más frecuentes: hipertensión arterial $(38,6 \%)$ hipotiroidismo (I5,8\%) y diabetes mellitus (II,9\%). El I3,9\% de los pacientes tuvo antecedente de neoplasia previa con compromiso en un $5 \%$ del tracto gastrointestinal como el sistema endocrino, $4 \%$ del tracto genitourinario, I \% de piel y sistema nervioso central.

\section{Características de los TNE}

a. Localización de TNE primarios y TNE metastásicos

$\mathrm{El}$ 59,4 \% de las muestras correspondieron a TNE primarios, de los cuales el $34,8 \%$ se ubicó en páncreas, $25,7 \%$ en intestino delgado y $\mathrm{I} 3,6 \%$ en estómago. Los menos frecuentes fueron aquellos ubicados en bronquios, endometrio, recto, tejidos blandos, válvula ileocecal y vesícula biliar, cada uno de los cuales correspondió al i \%.

El 40,6\% del total de las muestras, correspondió a pacientes con lesiones metastásicas, siendo la localización hepática la más frecuente con un $73,3 \%$ seguida del compromiso de ganglios linfáticos con $\mathrm{I} 5,5 \%$.

Un 93,7\% de las muestras analizadas correspondieron a TNE gastrointestinales o pancreáticos con una mayor proporción de localización hepática (tabla I).

\section{b. Clasificación OMS}

Un 71,2 \% de las muestras fue catalogado en el estudio histopatológico como bien diferenciado. De acuerdo con la clasificación empleada. El $27 \%$ de los TNE fueron tumores gastrointestinales o pancreáticos grado I, el $36 \%$ correspondieron al grado 2 y un $27,9 \%$ al grado 3 . El 3,6 \% correspondió a tumores pulmonares de grado bajo/ intermedio (carcinoide típico y atípico) y un 5,4 \% a tumores pulmonares de alto grado (células grandes y pequeñas).

\section{c. Marcadores tumorales}

El estudio de inmunohistoquímica se realizó de acuerdo con el cuadro clínico del paciente 
Tabla 1. Localización anatómica de las muestras de pacientes con tumores neuroendocrinos, analizadas en el servicio de patología de un hospital de alta complejidad de Medellín, 2010-2015.

\begin{tabular}{lc}
\hline Localización anatómica & $\mathbf{n}(\%)$ \\
\hline Hígado & $33(29,7)$ \\
Páncreas & $24(21,6)$ \\
Intestino delgado & $17(15,3)$ \\
Estómago & $9(8,1)$ \\
Ganglio linfático & $7(6,3)$ \\
Pulmón & $6(5,4)$ \\
Apéndice cecal & $3(2,7)$ \\
Hueso & $2(1,8)$ \\
Colon & $2(1,8)$ \\
Tejidos blandos & $2(1,8)$ \\
Bronquios & $1(0,9)$ \\
Endometrio & $1(0,9)$ \\
Esternón & $1(0,9)$ \\
Recto & $1(0,9)$ \\
Válvula ileocecal & $1(0,9)$ \\
Vesícula biliar & $1(0,9)$ \\
\hline
\end{tabular}

y las características morfológicas observadas al microscopio. La sinaptofisina fue reportada en IIO muestras con una positividad de $97,2 \%$ y la cromogranina se realizó en 99 muestras de las cuales el $87,8 \%$ fueron positivas. Por su parte el CD-56 se reportó en 58 muestras con un 9I,3\% de positividad y la citoqueratina (AEI/AE3) fue realizada en 60 muestras, con un resultado positivo del $95 \%$.

\section{Discusión}

En el presente estudio, el 93,7\% de las muestras correspondió a TNE gastrointestinales o pancreáticos, tanto primarios como metastásicos, lo cual es similar a lo publicado en la literatura ${ }^{8-9}$. En general, se ha descrito que el $25 \%$ de los tumores primarios del pulmón corresponden a TNE, incluso hay publicaciones que señalan que son más frecuentes que los TNE primarios localizados en el páncreas ${ }^{10}$. Esto contrasta con nuestros resultados, donde hay un claro predominio por los TNE pancreáticos primarios con respecto a los originados en el tracto respiratorio $(34,8 \%$ versus $9 \%$ respectivamente). Hay que tener en cuenta que la institución donde se realizó el estudio es un centro de referencia local para cirugía hepatobiliar y de páncreas, y esto puede explicar los resultados obtenidos.

En Estados Unidos, la incidencia de TNE es cercana a 5,86/10o ooo habitantes por año, y en general los tumores neuroendocrinos ocupan el $2 \%$ de todas las neoplasias ${ }^{\text {II }}$, con un ligero predominio en mujeres con respecto a los hombres $(2,5 / \mathrm{I})$, distinto a lo observado en nuestro estudio, donde no hubo un predominio por sexo.

En general, los TNE gastrointestinales o pancreáticos, pueden aparecer a cualquier edad. De acuerdo con las guías de la Sociedad Europea de Oncología Médica (ESMO, por su sigla en inglés), estas neoplasias tienen una mayor incidencia en la quinta década de la vida ${ }^{\text {Io }}$, lo cual es similar a nuestros resultados. Sin embargo, contrasta con una revisión norteamericana reciente de Oronsky et al. ${ }^{12}$, en la cual señalan la mayor frecuencia entre la sexta y séptima décadas. Es importante mencionar que, al referirse a tumores primarios del apéndice cecal, la edad de diagnóstico es significativamente menor y puede ubicarse entre los 20 y los 40 años ${ }^{\mathrm{II}, 13}$, como ocurrió en dos de los tres pacientes que fueron afectados con esta entidad en nuestro estudio, con edad al momento del diagnóstico de 34 y 32 años.

Con relación al uso de la inmunohistoquímica en el análisis de las muestras de tumores neuroendocrinos, vale la pena mencionar que, a nivel mundial se ha convertido en una herramienta supremamente útil en los departamentos de patología, dado que permite establecer el origen neuroendocrino de la muestra y así mismo ayuda en la subclasificación de la misma. En la actualidad, han sido descritos decenas de marcadores tumorales ${ }^{\mathrm{I}}$, sin embargo, los más usados en el caso de los TNE son la sinaptofisina (más sensible) y la cromogranina A (más específica). Los resultados del presente estudio concuerdan con lo reportado en la literatura mundial con respecto a la positividad de ambos marcadores en los casos de tumores neuroendocrinos ${ }^{15}$, sin embargo, como hallazgo adicional, se encontró que un alto porcentaje de las muestras también fue positiva para $\mathrm{CD}-56$ y $\mathrm{CK}(\mathrm{AEI} / \mathrm{AE} 3)$. 
En principio, el pilar del tratamiento de los pacientes con TNE es quirúrgico, logrando márgenes negativos con la resección completa del tumor ${ }^{16-17}$. No obstante, la aproximación terapéutica debe individualizarse teniendo en cuenta varios factores de la lesión como: el tamaño, el grado de diferenciación y la localización, entre otros. De igual manera, según lo descrito por varios autores ${ }^{11,16-18}$ se deben considerar aspectos del paciente como la edad y sus comorbilidades al momento de definir el manejo a realizar.

Debido a la rara presentación de estas neoplasias, no existen consensos internacionales que describan contraindicaciones absolutas para intervenir quirúrgicamente a los pacientes afectados por un tumor neuroendocrino. Aunque la mayoría de los casos evaluados en esta serie, tuvieron algún tipo de comorbilidad $(60,4 \%)$ no se conoce el grado de descompensación de estas enfermedades y, por lo tanto, no es posible identificar si fueron susceptibles de manejo quirúrgico al momento del diagnóstico.

Una de las limitaciones de este estudio es que fue realizado en una sola institución. Quizás este sea el primer estudio local que se realiza sobre las características de los pacientes con TNE (una enfermedad infrecuente), pero es posible que posteriormente se pueda ejecutar un análisis multicéntrico de esta patología, que permita identificar datos más reales de los pacientes afectados en la ciudad.

Por otra parte, se excluyeron registros médicos de pacientes cuyas muestras fueron enviadas al departamento de patología de nuestro hospital, pero en quienes el seguimiento clínico y el tratamiento se hizo en otra institución, lo cual no permitió seguir la evolución de los pacientes ni evaluar aspectos como la aparición de comorbilidades o neoplasias subsecuentes.

Durante la finalización del presente estudio, se dio a conocer una nueva propuesta de clasificación de la OMS para tumores pancreáticos neuroendocrinos ${ }^{19}$. Se analizaron los cambios más significativos de esta propuesta con respecto a lo establecido previamente por dicho organismo en 20Io, y se decidió continuar con la clasificación inicial, considerando que no se alteran los resultados de la investigación en cuanto a características clínicas e histopatológicas en la población estudiada.

Recientemente fue publicado un consenso entre la OMS y la IARC (International agency for research on cancer), que busca establecer una clasificación más uniforme en cualquier localización anatómica de los tumores neuroendocrinos. El objetivo de la clasificación es permitir una nomenclatura que correlacione factores genéticos y anatómicos. Se presentan algunas modificaciones de las definiciones tradicionales; entre ellas la definición de familia tumoral, dependiendo si se tratan de tumores pobremente diferenciados (carcinomas neuroendocrinos) y bien diferenciados (tumores neuroendocrinos); éstos últimos son divididos en grado I, 2 y 3 (con algunas excepciones como pulmón e hipófisis) con base en el conteo mitótico, índice de labilidad Ki-67 o presencia de necrosis.

Además, engloban en el término de neoplasias neuroendocrinas (NEN, por sus siglas en inglés) a todos los tumores de esta estirpe independiente de su grado de diferenciación. También denominan la variable "tipo tumoral" que se refiere a la familia más la adición del sitio anatómico del tumor (por ejemplo, NEC pulmonar tipo células pequeñas). Cabe destacar que las definiciones actuales de la OMS se mantendrán en la próxima edición de clasificación de tumores de esta entidad (WHO blue Book) en la cual se agregarán entre paréntesis las nuevas terminologías en los casos que difieran con las previas ${ }^{20}$.

\section{Conclusión}

Los TNE siguen siendo un grupo de neoplasias de poca frecuencia y de comportamiento clínico diverso. Las características clínicas e histopatológicas de los pacientes afectados en nuestra ciudad no difieren significativamente con los resultados de estudios similares realizados en otras latitudes. Los resultados presentados pueden servir como punto de partida a futuras investigaciones en nuestro medio, que relacionen dichas variables con otros desenlaces clínicos 
para determinar el pronóstico oncológico de los pacientes con esta entidad.

\section{Cumplimiento de normas éticas}

Consentimiento informado: El estudio fue avalado por el Comité de Investigaciones del Hospital Pablo Tobón Uribe y por el Comité de Ética de Investigación en Salud de la Universidad Pontificia Bolivariana. Por tratarse de un estudio basado en la revisión de informes de patología, esta investigación se considera de bajo riesgo de acuerdo con la Resolución 008430 de 1993 del Ministerio de Salud de Colombia, y no requirió el diligenciamiento de consentimiento informado por parte de los pacientes.

Conflicto de interés: ninguno declarado por los autores

Fuente de financiación: recursos propios de los autores.

\section{Referencias}

I. Schimmack S, Svejda B, Lawrence B, Kidd M, Modlin I. The diversity and commonalities of gastroenteropancreatic neuroendocrine tumors. Arch Surg. 201I;396:273298. https://doi.org/IO.IOO7/soo423-OII-O739-I

2. Vargas Martínez CC, Castaño Llano R. Tumores neuroendocrinos gastroenteropancreáticos. Rev Colomb Gastroenterol. 2010;25-2:165-176

3. Costa F, Domenichini E, Garavito G, Medrano R, Men$\operatorname{dez}$ G, O'Connor J, et al. Management of Neuroendocrine Tumors: A Meeting of Experts from Latin America. Neuroendocrinology. 2008;88:235-42. https://doi.org/Io.II59/oooI49356

4. Boushey RP, Dackiw APB. Carcinoid Tumors. Curr Treat Options Oncol. 2002;3:319-26. https://doi.org/Io.IO07/sII864-002-003I-2

5. Jernman J, Välimäki M, Louhimo J, Haglund C, Arola J. The Novel WHO 2010 Classification for Gastrointestinal Neuroendocrine Tumours Correlates Well with the Metastatic Potential of Rectal Neuroendocrine Tumours. Neuroendocrinology. 2012;95:317-24. https://doi.org/IO.II59/000333035

6. Hassan MMI, Phan A, Li D, Dagohoy CG, Leary C, Yao JC. Risk factors associated with neuroendocrine tumors: A U.S.-based case-control study. Int J Cancer. 2008;I23:867-73. https://doi.org/IO.IOO2/ijc.23529
7. Villa MG, Ronquillo CA, Moran AJ, Dip AK. Tumores neuroendocrinos: experiencia de 6 años en un centro de tercer nivel. Gaceta Mexicana de Oncología. 2015;I4:I4I-I49. https://doi.org/IO.IOI6/j.gamo.2015.07.003

8. National Comprehensive Cancer Network (NCCN). Clinical Practice Guidelines in Oncology: Neuroendocrine and Adrenal Tumors. Version I.20I8 - Fecha de consulta: 29 de marzo de 20I8. Disponible en: https:// www.nccn.org/professionals/physician_gls/default.aspx\#site

9. Karakus E, Helvacr A, Ekinci O, Dursun A. Comparison of WHO 2000 and WHO 2010 classiffications of gastroenteropancreatic neuroendocrine tumors. Turk J Gastroenterol 20I4;25:8I-7. https://doi.org/I0.5152/tjg.20I4.6579

Io. Oberg K, Knigge U, Kwekkeboom D. Perren A. Neuroendocrine gastro-entero-pancreatic tumors: ESMO Clinical Practice Guidelines for diagnosis, treatment and follow up. Ann Oncol. 2012;23 (Suppl.7vii):I24-30. https://doi.org/IO.I093/annonc/mds295

II. Alexandraki K, Karapanagioti A, Karoumpalis I, Bourzios G, Kaltsas G. Advances and Current Concepts in the Medical Management of Gastroenteropancreatic Neuroendocrine Neoplasms. BioMed Research International, 2017, Article ID 9856140, I2 pages. Fecha de consulta: 26 de abril de 2019 . https://doi.org/IO.II55/2017/9856I40

I2. Oronsky B, Ma PC, Morgensztern D, Carter CA. Nothing But NET: Review of Neuroendocrine Tumors and Carcinomas. Neoplasia. 2017;19:99I-IOO2. https://doi.org/Io.IoI6/j.neo.2017.09.002

I3. Florián MC, Uribe A, Cardona CP. Tumor carcinoide del apéndice cecal. Rev Colomb Cir. 20II;26:62-6.

I4. Duan K, Mete O. Algorithmic Approach to Neuroendocrine Tumors in Targeted Biopsies: Practical Applications of Immunohistochemical Markers. Cancer Cytopathol. 20I6;124:87I-84. https://doi.org/IO.IOO2/cncy.21765

I5. Yang Z, Klimstra DS, Hruban R, Tang L. Immunohistochemical Characterization of the Origins of Metastatic Well-differentiated neuroendocrine Tumors to the Liver. Am J Surg Pathol. 2017;4I:915-22. https://doi.org/I0.I097/PAS.00ooooooooooo876

I6. Raphael MJ, Chan DL, Law C, Singh S. Principles of diagnosis and management of neuroendocrine tumours. Canadian Med Associate J. 20I7;189:E398-E404. https://doi.org/I0.I503/cmaj.I6077I

I7. Farley HA, Pommier RF. Surgical Treatment of Small Bowel Neuroendocrine Tumors. Hematol Oncol Clin N Am. 20I6;30:49-6I.

https://doi.org/IO.IOI6/j.hoc.2015.09.00I 
I8. Tamburrino D, Spoleti G, Partelli S, Muffatti F, Adamenko O, Crippa $S$ et al. Surgical management of neuroendocrine tumors. Best Pract Res Clin Endocrinol Metab. 2016;30:93-IO2.

https://doi.org/IO.IOI6/j.beem.2015.10.003

19. Klöppel G, Klimstra D, Hruban R, Adsay V, Capella C, Couvelard A, et al. Pancreatic Neuroendocrine Tumors: Update on the New World Health Organization Classification. AJSP: Reviews \& Reports. 2017;22:233-9. https://doi.org/I0.I097/PCR.00000000000002II
20. Rindi G, Klimstra DS, Abedi-Ardekani B, Asa SL, Bosman FT, Brambilla E, et al. A common classification framework for neuroendocrine neoplasms: an International Agency for Research on Cancer (IARC) and World Health Organization (WHO) expert consensus proposal. Modern Pathology. 2018;31:1770-86. https://doi.org/IO.I038/s4I379-0I8-OIIO-y 